\title{
Estudo da vazão de uma fonte por meio da videoanálise: uma proposta uti- lizando recipientes na forma de prismas regulares ${ }^{+*}$
}

\author{
Silva, E. S. ${ }^{1}$ \\ Lima, A. R. ${ }^{2}$ \\ Instituto Federal de Educação, Ciência e Tecnologia de Mato Grosso \\ Campus Juína - MT
}

\begin{abstract}
Resumo
O trabalho discute os resultados de uma videoanálise utilizada para investigar a vazão de uma fonte de água que alimenta diferentes recipientes, na forma de prismas regulares. A investigação proposta pode ser utilizada para trabalhar técnicas de modelagem matemática em disciplinas de física básica. Os resultados revelam que o modelo de investigação por videoanálise permite obter a vazão esperada para a fonte com desvio percentual de até 4\%. A ideia apresentada pode ser utilizada para o estudo da vazão de fontes em aplicações hidrológicas diversas, considerando o volume de água que se acumula em recipientes que podem ser aproximados por prismas regulares.
\end{abstract}

Palavras-chave: Vazão; Videoanálise; Modelagem Matemática; Prismas Regulares.

\begin{abstract}
This paper discusses the results of an analytical research to investigate a flow of a water source that feeds different containers in the form of regular prisms. The research can be used for the treatment of mathematical modeling techniques in basic physics disciplines. The results show that the video research model allows obtaining an expected flow rate for a source with a percentage deviation of up to $4 \%$. The idea presented can be used
\end{abstract}

\footnotetext{
+ Study of the flow of a source through video analysis: a proposal using containers in the form of regular prisms

* Recebido: maio de 2017. Aceito: agosto de 2017.

${ }^{1}$ E-mail: ssf.erick@gmail.com, ${ }^{2}$ E-mail: andre.rubens.7@gmail.com
} 
for the study of the flow of sources in hydrological applications, considering the volume of water that accumulates in containers that can be approximated by regular prisms.

Keywords: Flow; Video Analysis; Mathematical Modeling; Regular Prisms.

\section{Introdução}

A dinâmica de fluidos fornece ferramentas para a modelagem matemática que vão além do trabalho envolvendo equilíbrio de colunas líquidas. A literatura recente relata aplicações em situações práticas como o estudo do tráfego de veículos (BOGO; GRAMANI; KAVISKI, 2015), modelagem do fluxo de pessoas em cidades e vias urbanas (VARGAS; GRAMANI; KAVISKI; BALBO, 2012), utilização de recursos computacionais para analisar a transferência de calor (ROCHA; SILVEIRA, 2012; SILVA; SOUZA; MARQUES, 2009), investigação de propriedades dinâmicas de líquidos e grãos (LÓPEZ; DEMARCHI; AKAMATSU; VERCIK, 2010; LÓPEZ; PASTORELLO; ARCE, 2014; LINARES; PASTORELLO; ARCE; 2012) e, até mesmo, o estudo de sistemas biológicos, como o fluxo de sangue no corpo humano (VARGAS; ARGENTA, 2015). Outros trabalhos no contexto do ensino de física discutem propriedades hidrostáticas e hidrodinâmicas, como a velocidade de escoamento horizontal da água em ductos e a sustentação da asa de aviões (LOPES, 2010; WELTNER et al., 2001). Neste artigo é apresentada uma forma de estudar a vazão de uma determinada fonte de água, a partir da filmagem do processo de escoamento em recipientes que possam ser aproximados por prismas regulares. Frequentemente, medidas de vazões, níveis de água e outros parâmetros de interesse hidrométricos, que permitem estudar fontes de água encontradas na natureza, são requeridos. O monitoramento dessas grandezas possibilita uma melhoria na eficiência e minimização de custos em projetos nas áreas de ciências, engenharia e aplicações tecnológicas correlatas.

A vazão da fonte foi investigada no presente trabalho por meio da videoanálise. Essa técnica permite investigar fenômenos físicos a partir de suas filmagens (BEICHNER, 1996; LAWS; PFISTER, 1998; BROWN; COX, 2009; BEZERRA et al., 2012; LENZ et al., 2014; DE JESUS, 2014; BEZERRA; LENZ; SAAVEDRA, 2016). A velocidade vertical da coluna líquida, que aumenta no interior do recipiente de volume $V$, será relacionada com a vazão $Z$ por meio de um modelo matemático válido para recipientes na forma de prismas regulares. $\mathrm{O}$ valor da vazão obtida por meio da videoanálise é comparado com a vazão esperada para a fonte, que pode ser estimada a partir de um recipiente de volume conhecido e um cronômetro. A comparação desses valores permite avaliar se o modelo de investigação proposto é válido, para descrever o comportamento do volume no interior do recipiente em função do tempo, e, consequentemente, da vazão associada à fonte. 


\section{Modelo para estudar a vazão utilizando recipientes na forma de prismas regulares}

A vazão de uma fonte pode ser obtida por meio da videoanálise, estudando a velocidade de subida da coluna líquida no interior de um recipiente com volume conhecido. Por exemplo, quando uma torneira é mantida aberta, a água vai preenchendo o recipiente de volume $V$, ao longo de um intervalo de tempo $\Delta t$. A razão entre a variação de volume $\Delta V$ do líquido no recipiente e o intervalo de tempo $\Delta t$ associado, fornece a vazão $Z$, suposta constante (ÇENGEL; CIMBALA, 2007; LEITHOLD, 1994).

No entanto, a velocidade com que a água sobe depende da geometria do reservatório. Neste trabalho, são discutidos recipientes que possam ser aproximados por prismas regulares. Inicialmente, dois prismas $\mathrm{P}_{1}$ e $\mathrm{P}_{2}$, com área de seção transversal constante ao longo da altura $h$, são investigados. Em seguida, é analisado um sólido $\mathrm{P}_{3}$, que pode ser dividido em três prismas regulares na forma de paralelepípedos retângulos, enfatizando a ideia de se aproximar volumes não usuais por volumes conhecidos, quando possível. A Fig. 1 apresenta os três recipientes utilizados com volumes denotados por $V_{1}, V_{2}$ e $V_{3}$ (Fig.1).

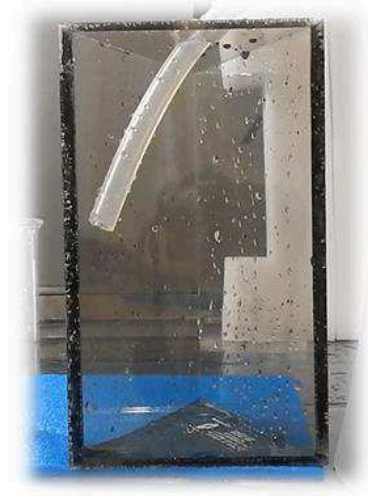

(a)

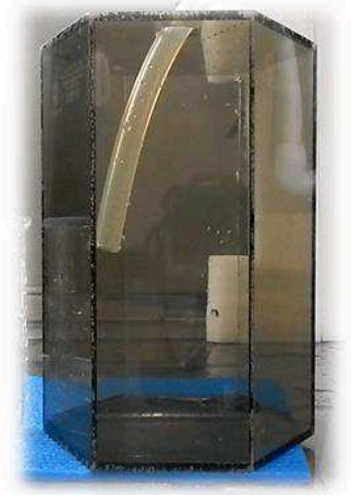

(b)

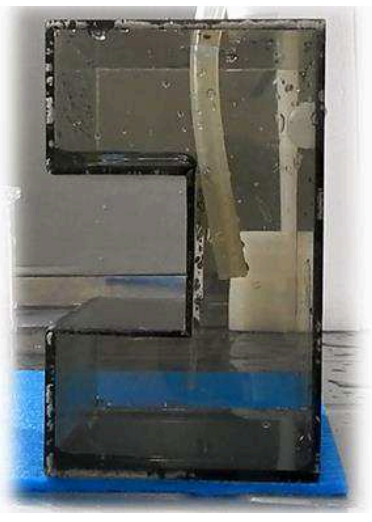

(c)

Fig. 1 -Recipientes utilizados para estudar a vazão $Z$ de uma fonte de água.

Observe que o volume de um prisma regular, cuja área da seção transversal se mantém constante ao longo da vertical, pode ser obtido a partir da expressão

$$
V=A_{b} h \text {, }
$$

na qual $A_{\mathrm{b}}$ e $h$ representam a área da base e a altura do prisma, respectivamente. Vale notar que a área de um polígono regular com $n$ lados, sendo que cada um deles mede $a$, pode ser expressa por

$$
A_{b}=\frac{1}{2} \text { nam }
$$

na qual $m$ representa o apótema dos $n$ triângulos equiláteros nos quais a base foi dividida. Dessa maneira, o volume do prisma regular pode ser obtido pela equação 


$$
V=\frac{1}{2} n a m h
$$

Uma vez determinado o volume do prisma regular, pode-se diferenciá-lo em relação ao tempo, obtendo a seguinte expressão para a vazão

$$
Z=\frac{d V}{d t}=\frac{1}{2} \operatorname{nam} \frac{d h}{d t},
$$

sendo $\frac{d h}{d t}$ a velocidade de subida da coluna líquida, que será estudada por meio da videoanálise (ÇENGEL; CIMBALA, 2007; LEITHOLD, 1994).

\section{II.1 Vazão estudada a partir de um reservatório em forma de prisma regular com base triangular $\mathbf{R}_{1}$}

Seja um prisma regular de base triangular, com os lados de sua base medindo $a$ (Fig. 1a). A expressão (3) pode ser utilizada para a obtenção do seu volume

$$
V_{1}=\frac{\sqrt{3}}{4} a^{2} h,
$$

uma vez que $n=1$ e $m=\frac{a \sqrt{3}}{2}$, sendo $h$ a altura do prisma.

Perceba que a expressão (4) fornece a vazão $Z_{1}$ da fonte que alimenta o recipiente $\mathrm{R}_{1}$, em função da velocidade vertical da coluna líquida. Com efeito, fazendo $n=1$ e $m=\frac{a \sqrt{3}}{2}$, temse

$$
Z_{1}=\frac{d V_{1}}{d t}=\frac{\sqrt{3}}{4} a^{2} \frac{d h}{d t}
$$

em que $\frac{d h}{d t}$ representa a velocidade vertical da coluna líquida no interior do recipiente $\mathrm{R}_{1}$.

\section{II.2 Vazão estudada a partir de um reservatório em forma de prisma regular com base hexagonal $\mathbf{R}_{2}$}

Seja um prisma regular de base hexagonal, com as arestas da base medindo $a$ (Fig. 1b). $O$ volume $V_{2}$ desse prisma também pode ser obtido por meio da expressão (3)

$$
V_{2}=\frac{1}{2} n a m \frac{d h}{d t}=\frac{3 \sqrt{3}}{2} a^{2} h,
$$

uma vez que $n=6$ e $m=\frac{a \sqrt{3}}{2}$. 
A expressão (4) permite obter a vazão $Z_{2}$ da fonte que alimenta o recipiente $\mathrm{R}_{2}$, em função da velocidade de subida da coluna líquida. De fato, fazendo $n=6$ e $m=\frac{a \sqrt{3}}{2}$, tem-se

$$
Z_{2}=\frac{d V_{2}}{d t}=\frac{3 \sqrt{3}}{2} a^{2} \frac{d h}{d t}
$$

\section{II.3 Vazão estudada a partir de um reservatório não usual $\mathbf{R}_{3}$}

Seja um sólido não usual $\mathrm{R}_{3}$, conforme indica a figura 1c (Fig.1c). Ele pode ser dividido em três prismas regulares na forma de paralelepípedos retangulares $p_{1}, p_{2}$ e $p_{3}$. O volume de um paralelepípedo retangular $p$ é dado pelo produto da área da base com sua respectiva altura. Considerando os lados da base como sendo $a$ e $b$, o volume do paralelepípedo $V_{\mathrm{p}}$ fica determinado pela equação

$$
V_{p}=a b h
$$

sendo $h$ a altura do paralelepípedo retangular.

A expressão (9) permite obter a vazão $Z_{p}$ da fonte, em função da velocidade de subida da coluna líquida ao longo do paralelepípedo $p$. De fato, diferenciando (9) com respeito ao tempo $t$, tem-se

$$
Z_{p}=\frac{d V_{p}}{d t}=a b \frac{d h}{d t}
$$

com $\frac{d h}{d t}$ representando a velocidade vertical da coluna líquida no interior do paralelepípedo $p$ (ÇENGEL; CIMBALA, 2007; LEITHOLD, 1994).

\section{Modelo de escoamento a partir da videoanálise}

A vazão da fonte que alimenta um recipiente pode ser expressa em função da velocidade vertical da coluna líquida, que aumenta no interior do reservatório ao longo do tempo. A videoanálise permite estudar a relação entre a posição $h$ dessa coluna líquida e o tempo $t$, quadro a quadro (Fig. 2). O software Tracker foi utilizado para analisar o arquivo em formato de vídeo do escoamento investigado (TRACKER, 2017). Trata-se de um software livre concebido para utilização no ensino de física, sendo ligado ao projeto Open Source Physics (BROWN; COX, 2009). Observe que, como os recipientes estudados possuem área da seção transversal constante ao longo da vertical, pode-se modelar a posição vertical da coluna de água por meio de uma função afim do tipo $h(t)=v t+h_{0}$ (Fig. 3). Até mesmo o reservatório não usual $\mathrm{R}_{3}$ apresenta seções transversais constantes $A_{1}, A_{2}$ e $A_{3}$, considerando três alturas correspondentes $h_{1}, h_{2}$ e $h_{3}$. A partir de um ajuste linear dos pontos $h$ e $t$, pode-se obter o coeficiente angular $v$, ou seja, a velocidade vertical da coluna líquida no interior do reservatório (Tabela 1). Todo o material 
utilizado no presente trabalho encontra-se disponível em: <https://drive.google.com/open?id=0B0S3ARHrAIgPQklnN2Exd3NtRHM>.
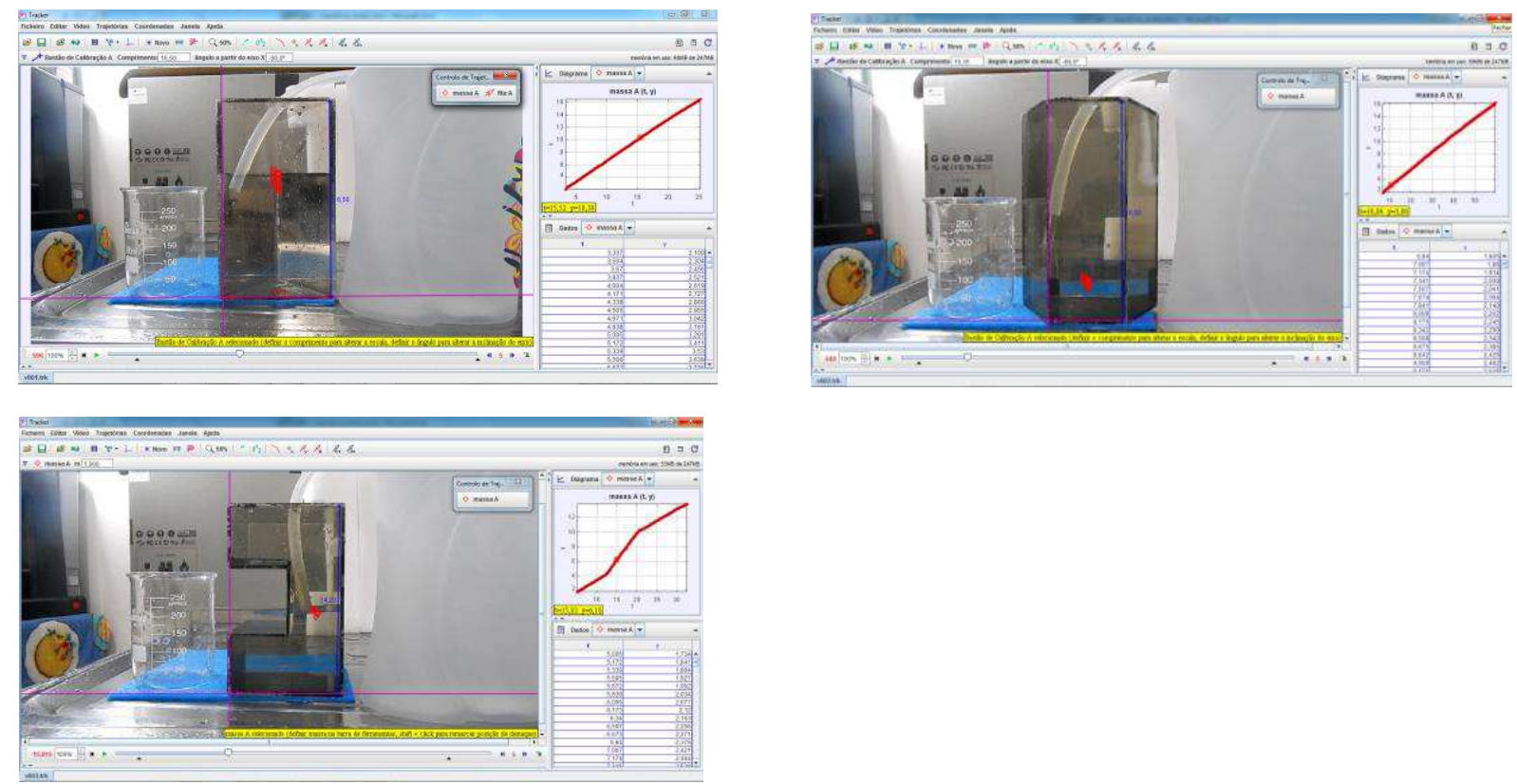

Fig. 2 - Tela do Tracker mostrando os experimentos realizados com os diferentes recipientes $R_{1}, R_{2}$ e $R_{3}$.
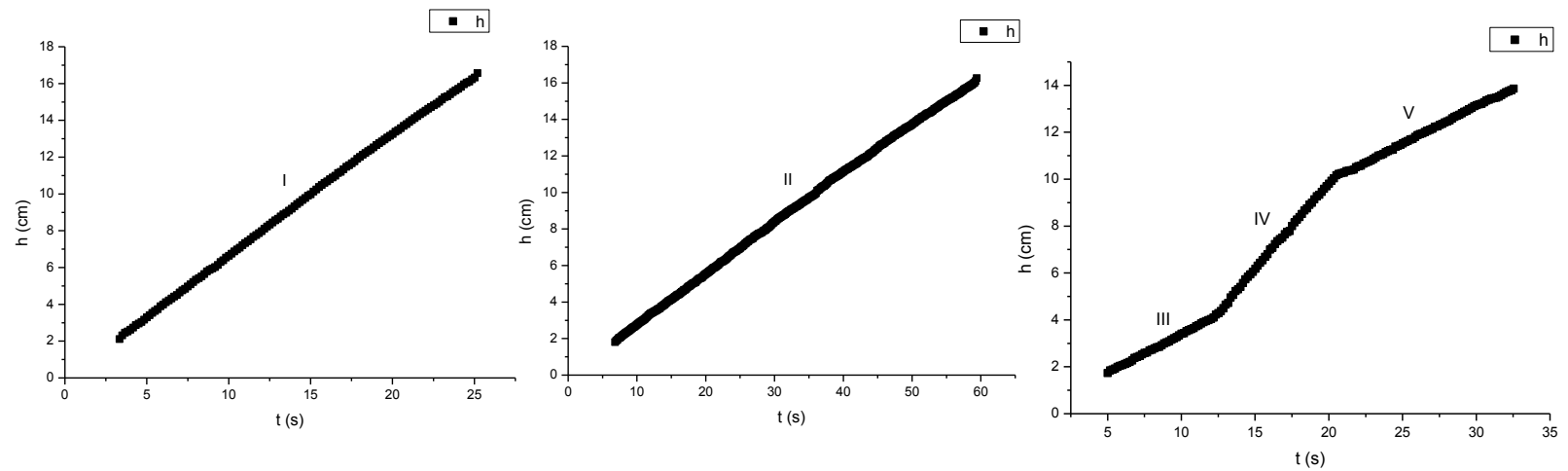

Fig. 3 - Relação entre he t obtida a partir da filmagem da vazão de uma torneira aberta e conectada por um ducto aos reservatórios $R_{1}, R_{2}$ e $R_{3}$.

Tabela 1 - Velocidade da coluna líquida nos trechos I, II, III, IV e V da Fig. 3, obtida por meio de um ajuste linear do tipo $h(t)=v t+h_{0}$.

\begin{tabular}{|c|c|}
\hline Trecho & $\boldsymbol{v}(\mathbf{c m} / \mathbf{s})$ \\
\hline I & $0,6539 \pm 0,0009$ \\
\hline II & $0,2736 \pm 0,0003$ \\
\hline III & $0,3308 \pm 0,0435$ \\
\hline IV & $0,7373 \pm 0,0030$ \\
\hline V & $0,3169 \pm 0,0011$ \\
\hline
\end{tabular}




\section{Resultados e discussões}

A videoanálise permite obter o valor da velocidade vertical da coluna líquida que aumenta no interior do reservatório ao longo do tempo. Uma vez obtida a velocidade da coluna líquida, pode-se calcular a vazão $Z$ associada. A tabela seguinte (Tabela 2) apresenta a comparação da vazão obtida por meio da videoanálise com a vazão esperada para a fonte. A vazão esperada $Z_{0}$ foi determinada medindo o volume $\Delta V$ de água que deixa a fonte no intervalo de tempo $\Delta t$. Isso pode ser realizado, por exemplo, com um béquer de $250 \mathrm{~mL}$ e um cronômetro. Observe que os desvios percentuais em relação à vazão esperada são menores ou iguais do que $4 \%$.

Tabela 2 - Vazão de referência $Z_{0}$, vazão $Z$ obtida pelo modelo e o erro percentual associado $\varepsilon$.

\begin{tabular}{|c|c|c|c|}
\hline Reservatório & $\boldsymbol{Z}_{\mathbf{0}}\left(\mathbf{m}^{3} / \mathbf{s}\right)$ & $\boldsymbol{Z}\left(\mathbf{m}^{3} / \mathbf{s}\right)$ & $\boldsymbol{\varepsilon} \%$ \\
\hline $\mathrm{R}_{1}$ & $2,24 \times 10^{-5}$ & $2,26 \times 10^{-5}$ & $1 \%$ \\
\hline $\mathrm{R}_{2}$ & $2,30 \times 10^{-5}$ & $2,39 \times 10^{-5}$ & $4 \%$ \\
\hline $\mathrm{R}_{3 \mathrm{a}}$ & $2,41 \times 10^{-5}$ & $2,32 \times 10^{-5}$ & $4 \%$ \\
\hline $\mathrm{R}_{3 \mathrm{~b}}$ & $2,45 \times 10^{-5}$ & $2,38 \times 10^{-5}$ & $3 \%$ \\
\hline $\mathrm{R}_{3 \mathrm{c}}$ & $2,29 \times 10^{-5}$ & $2,22 \times 10^{-5}$ & $3 \%$ \\
\hline
\end{tabular}

\section{Considerações finais}

O trabalho apresenta uma maneira de investigar a vazão de uma fonte por meio da videoanálise. Basta a obtenção de um filme da referida fonte alimentando um recipiente de volume $V$, com pelo menos uma aresta conhecida. Esse filme é aberto no programa Tracker, que permite auferir a relação entre a posição vertical da coluna líquida $h$ e o tempo $t$. A partir de um ajuste linear, pode-se obter a velocidade de subida da coluna líquida que se acumula no interior do reservatório. Conhecendo-se essa velocidade vertical da coluna, obtém-se a vazão $Z$ da fonte. Esse resultado pode ser comparado com a vazão $Z_{0}$ esperada. No presente trabalho, a videoanálise permitiu obter a vazão $Z$ da fonte investigada com erros percentuais de até $4 \%$, em relação à vazão esperada $Z_{0}$. Isso permite apontar para a validade do modelo adotado, que pode ser utilizado para trabalhar técnicas de modelagem matemática em disciplinas de física básica, além de auxiliar na estimativa de parâmetros hidrométricos de interesse científico.

\section{Referências}

BEICHNER, R. The impact of video motion analysis on kinematics graph interpretation skills. American Journal of Physics, v. 64, p. 1272-1277, 1996. 
BEZERRA Jr, A G.; OLIVEIRA, L. P.; LENZ, J. A.; SAAVEDRA, N. Videoanálise com o software livre Tracker no laboratório didático de Física: movimento parabólico e segunda lei de Newton. Caderno Brasileiro de Ensino de Física, v. 29, n. Especial 1, p. 469-490, 2012. BEZERRA, A. G.; LENZ, J. A.; FILHO, N. C. S. Acta Scientiae, v. 18, n. 3, p. 782-798, 2016. BOGO, R. L.; GRAMANI, L. M.; KAVISKI, E. Modelagem computacional do tráfego de veículos pela teoria microscópica. Revista Brasileira de Ensino de Física, v. 37, n. 1, p. 1301, 2015.

BROWN, D.; COX, A. J. Innovative Uses of Video Analysis. The Physics Teacher, v. 47, p. 328, 2009.

ÇENGEL, Y. A.; CIMBALA, J. M. Mecânica dos fluidos: Fundamentos e aplicações. São Paulo: McGraw-Hill, 2007.

DE JESUS, V. L. B. Experimentos e videoanálise: Dinâmica. São Paulo: Livraria da Física, 2014.

LAWS, P.; PFISTER, H. Using digital video analysis in introductory mechanics projects. The Physics Teacher, v. 36, p. 282-287, 1998.

LEITHOlD, L. O Cálculo com Geometria Analítica. 3 ed. São Paulo: Editora Harbra Ltda, 1994. v. 2.

LENZ J. A.; FILHO, N. C. S.; BEZERRA Jr, A. G. Abakós, Belo Horizonte, v. 2, n. 2, p. 2434, 2014.

LINARES, J. L.; PASTORELlO, I. M.; ARCE, A. I. C. Vazão de grãos em um silo cilíndrico: uma atividade experimental. Revista Brasileira de Ensino de Física, v. 34, n. 4, p. 4308, 2012.

LOPES, W. Velocidade de escoamento horizontal de água por um conduto. Caderno Brasileiro de Ensino de Física, v. 27, n. 2, p. 385-393, 2010.

LÓPEZ, J.; DEMARCHI, T.; AKAMATSU, M.; VERCIK, A. Estudo comparativo de algumas propriedades dinâmicas de líquidos e grãos na sala de aula. Revista Brasileira de Ensino de Física, v. 32, n. 1, p. 1301, 2010.

LÓPEZ, J.; PASTORELLO, I. M.; ARCE, A. I. C. Vazão facilitada de grãos de arroz de um silo cilíndrico usando "obstruções" esféricas ou cônicas. Revista Brasileira de Ensino de Física, v. 36, n. 1, p. 1303, 2014.

ROCHA, P. A. C.; SILVEIRA, J. V. P. Estudo e aplicação de simulação computacional em problemas simples de mecânica dos fluidos e transferência de calor. Revista Brasileira de Ensino de Física, v. 34, n. 4, p. 4306, 2012. 
SILVA, E. L.; SOUZA, A. R.; MARQUES, E. M. R. Alguns estudos de fluxo de fluido utilizando software gráfico. Revista Brasileira de Ensino de Física, v. 31, n. 3, p. 3502, 2009.

VARGAS, M.; ARGENTA, M. A. Uma representação do fluxo sanguíneo pulsátil em artérias ou veias usando lattice Boltzmann. Revista Brasileira de Ensino de Física, v. 37, n. 4, p. 4304, 2015.

VARGAS, M.; GRAMANI, L. M.; KAVISKI, E.; BALBO, F. A. Modelagem do fluxo de pedestres pela teoria macroscópica. Revista Brasileira de Ensino de Física, v. 34, n. 4, p. 4318, 2012 .

WELTNER, K.; SUNDBERG, M. I.; ESPERIDIÃO, A. S.; MIRANDA, P. A dinâmica dos fluidos complementada e a sustentação da asa. Revista Brasileira de Ensino de Física, v. 23, n. 4, dezembro, 2001.

TRACKER. Site do software Tracker. Disponível em: <http://physlets.org/tracker>. Acesso em: 26 mai. 2017. 